\title{
Oscillation of strongly noncanonical equations
}

\section{Blanka Baculikova}

Department of Mathematics, Faculty of Electrical Engineering and Informatics, Technical University of Košice, Letná 9, 04200 Košice, Slovakia.

\begin{abstract}
New oscillation criteria for third order noncanonical differential equations of the form

$$
\left(r_{2}(t)\left(r_{1}(t) y^{\prime}(t)\right)^{\prime}\right)^{\prime}+p(t) y(\tau(t))=0
$$

are established. Our technique employs an equivalent canonical representation of the studied equation, which essentially simplifies the examination of noncanonical equations. The results obtained are supported by several illustrative examples.
\end{abstract}

Keywords: Oscillation, third order differential equations, noncanonical operator.

2010 MSC: 34C10, 34K11.

(C)2018 All rights reserved.

\section{Introduction}

We consider third order linear delay differential equations of the form

$$
\left(r_{2}(t)\left(r_{1}(t) y^{\prime}(t)\right)^{\prime}\right)^{\prime}+p(t) y(\tau(t))=0
$$

where

$\left(H_{1}\right) r_{i} \in C^{(3-i)}\left(t_{0}, \infty\right), r_{i}(t)>0, i=1,2 ;$

$\left(\mathrm{H}_{2}\right) \mathrm{p} \in \mathrm{C}\left(\mathrm{t}_{0}, \infty\right), \mathrm{p}(\mathrm{t}) \geqslant 0$;

$\left(H_{3}\right) \tau \in C^{1}\left(\left[t_{0}, \infty\right)\right), \tau^{\prime}(t) \geqslant 0, \tau(t) \leqslant t, \lim _{t \rightarrow \infty} \tau(t)=\infty$.

In the sequel, we assume that (E) is strongly noncanonical, that is,

$$
\int^{\infty} \frac{1}{r_{i}(s)} d s<\infty, \quad i=1,2 .
$$

By a solution of Eq. (E) we mean a function $y(t) \in C^{3}\left(\left[T_{x}, \infty\right)\right), T_{x} \geqslant t_{0}$, which satisfies Eq. (E) on

Email address: blanka.baculikova@tuke.sk (Blanka Baculikova)

doi: $10.22436 /$ jnsa.011.10.02

Received: 2018-04-11 Revised: 2018-06-14 Accepted: 2018-06-19 
$\left[T_{x}, \infty\right)$. We consider only those solutions $y(t)$ of $(E)$, which satisfy $\sup \{|y(t)|: t \geqslant T\}>0$ for all $T \geqslant T_{x}$. We assume that (E) possesses such a solution. A solution of (E) is called oscillatory if it has arbitrarily large zeros on $\left[T_{x}, \infty\right)$, and otherwise it is called to be nonoscillatory. Equation (E) is said to be oscillatory if all its solutions are oscillatory.

There are numerous papers dealing with oscillatory properties of (E) (see, e.g., $[1-8,10])$. Most papers are devoted to canonical equations, where conditions opposite to (1.1) are assumed to hold, that is,

$$
\int^{\infty} \frac{1}{r_{i}(s)} d s=\infty, \quad i=1,2
$$

The reason is that the examination of canonical equation is much simpler. In this paper, we connect theory of noncanonical equations with that of canonical equations.

When considering nonoscillatory solutions of $(\mathrm{E})$, we can restrict our attention to positive ones. It follows from a generalization of lemma of Kiguradze (see, e.g., $[1,4,5]$ ) that the set of positive solutions of $(E)$ has the following structure.

Lemma 1.1. Assume that $\mathrm{y}(\mathrm{t})$ is a positive solution of $(\mathrm{E})$, then $\mathrm{y}(\mathrm{t})$ satisfies one of the following conditions

$$
\begin{array}{lll}
r_{1}(t) y^{\prime}(t)<0, & r_{2}(t)\left(r_{1}(t) y^{\prime}(t)\right)^{\prime}>0, & \left(r_{2}(t)\left(r_{1}(t) y^{\prime}(t)\right)^{\prime}\right)^{\prime}<0, \\
r_{1}(t) y^{\prime}(t)<0, & r_{2}(t)\left(r_{1}(t) y^{\prime}(t)\right)^{\prime}<0, & \left(r_{2}(t)\left(r_{1}(t) y^{\prime}(t)\right)^{\prime}\right)^{\prime}<0, \\
r_{1}(t) y^{\prime}(t)>0, & r_{2}(t)\left(r_{1}(t) y^{\prime}(t)\right)^{\prime}<0, & \left(r_{2}(t)\left(r_{1}(t) y^{\prime}(t)\right)^{\prime}\right)^{\prime}<0, \\
r_{1}(t) y^{\prime}(t)>0, & r_{2}(t)\left(r_{1}(t) y^{\prime}(t)\right)^{\prime}>0, & \left(r_{2}(t)\left(r_{1}(t) y^{\prime}(t)\right)^{\prime}\right)^{\prime}<0 .
\end{array}
$$

So, if we are about to establish oscillatory criterion for strongly noncanonical equation, we have to eliminate four above mentioned classes which may lead to four independent conditions. To avoid this, we present a simple condition that yields to a canonical representation of $(E)$, which essentially simplifies examination of $(\mathrm{E})$.

\section{Main results}

Throughout the paper, we assume that (1.1) holds and so we can employ the notation

$$
\pi_{1}(\mathrm{t})=\int_{\mathrm{t}}^{\infty} \frac{1}{r_{1}(s)} \mathrm{ds}, \quad \pi_{2}(\mathrm{t})=\int_{\mathrm{t}}^{\infty} \frac{1}{\mathrm{r}_{2}(\mathrm{~s})} \mathrm{ds}, \quad \pi(\mathrm{t})=\int_{\mathrm{t}}^{\infty} \frac{1}{\mathrm{r}_{1}(\mathrm{~s})} \int_{s}^{\infty} \frac{1}{\mathrm{r}_{2}(u)} \mathrm{duds}
$$

Theorem 2.1. Assume that

$$
\frac{r_{1}(t) \pi_{1}(t)}{r_{2}(t) \pi_{2}(t)}=k, \quad k>0
$$

Then strongly noncanonical differential equation (E) can be written in canonical form as

$$
\frac{1}{\pi(t)}\left(r_{2}(t) \pi(t)\left(r_{1}(t) \pi(t)\left(\frac{y(t)}{\pi(t)}\right)^{\prime}\right)^{\prime}\right)^{\prime}+p(t) y(\tau(t))=0
$$

Proof. Direct computation reveals that

$$
r_{2}(t) \pi(t)\left(r_{1}(t) \pi(t)\left(\frac{y(t)}{\pi(t)}\right)^{\prime}\right)^{\prime}=r_{2}(t)\left(r_{1}(t) y^{\prime}(t)\right)^{\prime} \pi(t)+r_{2}(t) y^{\prime}(t) \pi_{2}(t)-y(t)+y(t) \frac{r_{2}(t) \pi_{2}^{2}(t)}{r_{1}(t) \pi(t)} .
$$

It follows from (2.1) that

$$
\frac{\pi_{1}(\mathrm{t}) \pi_{2}(\mathrm{t})}{\pi(\mathrm{t})}=1+\mathrm{k}
$$


Setting into (2.2) one gets

$$
r_{2}(t) \pi(t)\left(r_{1}(t) \pi(t)\left(\frac{y(t)}{\pi(t)}\right)^{\prime}\right)^{\prime}=r_{2}(t)\left(r_{1}(t) y^{\prime}(t)\right)^{\prime} \pi(t)+\frac{1}{k} r_{1}(t) y^{\prime}(t) \pi_{1}(t)+\frac{1}{k} y(t) .
$$

Therefore,

$$
\frac{1}{\pi(t)}\left(r_{2}(t) \pi(t)\left(r_{1}(t) \pi(t)\left(\frac{y(t)}{\pi(t)}\right)^{\prime}\right)^{\prime}\right)^{\prime}=\left(r_{2}(t)\left(r_{1}(t) y^{\prime}(t)\right)^{\prime}\right)^{\prime},
$$

which yields that equations $(E)$ and $\left(E^{*}\right)$ are equivalent.

Now we shall show that $\left(E^{*}\right)$ is canonical, that is,

$$
\int^{\infty} \frac{1}{r_{1}(s) \pi(s)} d s=\int^{\infty} \frac{1}{r_{2}(s) \pi(s)} d s=\infty
$$

It is easy to see that

$$
\int_{\mathrm{t}_{0}}^{\infty} \frac{1}{r_{1}(s) \pi(s)} \mathrm{d} s=\int_{\mathrm{t}_{0}}^{\infty} \frac{\pi_{2}(s)}{r_{1}(s) \pi(s)} \frac{1}{\pi_{2}(s)} \mathrm{d} s \geqslant \frac{1}{\pi_{2}\left(\mathrm{t}_{0}\right)} \int_{\mathrm{t}_{0}}^{\infty} \frac{-\pi^{\prime}(s)}{\pi(s)} \mathrm{d} s=\infty
$$

The second integral can be evaluated similarly and the proof is complete.

Corollary 2.2. Let (2.1) holds. If $\mathrm{y}(\mathrm{t})$ is a positive solution of $(\mathrm{E})$, then $z(t)=y(t) / \pi(t)$ is a positive solution of

$$
\left(\mathrm{r}_{2}(\mathrm{t}) \pi(\mathrm{t})\left(\mathrm{r}_{1}(\mathrm{t}) \pi(\mathrm{t}) z^{\prime}(\mathrm{t})\right)^{\prime}\right)^{\prime}+\pi(\mathrm{t}) \pi(\tau(\mathrm{t})) \mathrm{p}(\mathrm{t}) z(\tau(\mathrm{t}))=0
$$

and $z(t)$ satisfies one of the following conditions

$$
\begin{array}{lll}
z^{\prime}(\mathrm{t})<0, & \left(\mathrm{r}_{1}(\mathrm{t}) \pi(\mathrm{t}) z^{\prime}(\mathrm{t})\right)^{\prime}>0, & \left(\mathrm{r}_{2}(\mathrm{t}) \pi(\mathrm{t})\left(\mathrm{r}_{1}(\mathrm{t}) \pi(\mathrm{t}) z^{\prime}(\mathrm{t})\right)^{\prime}\right)^{\prime}<0 \\
z^{\prime}(\mathrm{t})>0, & \left(\mathrm{r}_{1}(\mathrm{t}) \pi(\mathrm{t}) z^{\prime}(\mathrm{t})\right)^{\prime}>0, & \left(\mathrm{r}_{2}(\mathrm{t}) \pi(\mathrm{t})\left(\mathrm{r}_{1}(\mathrm{t}) \pi(\mathrm{t}) z^{\prime}(\mathrm{t})\right)^{\prime}\right)^{\prime}<0 .
\end{array}
$$

Corollary 2.2 simplifies structure of possible positive solutions of $(\mathrm{E})$. To establish oscillatory criterion for $(E)$ it is sufficient to eliminate the classes $\left(N_{0}\right)$ and $\left(N_{2}\right)$ instead of four classes presented in Lemma 1.1 .

Theorem 2.3. Let (2.1) holds. Assume that $\mathrm{y}(\mathrm{t})$ is a positive solution of $(\mathrm{E})$. If

$$
\limsup _{t \rightarrow \infty} \int_{\tau(t)}^{t} \frac{1}{r_{1}(v) \pi(v)} \int_{v}^{t} \frac{1}{r_{2}(u) \pi(u)} \int_{u}^{t} \pi(x) \pi(\tau(x)) p(x) d x d u d v>1,
$$

then the corresponding $z(t)=y(t) / \pi(t)$ does not satisfy $\left(\mathrm{N}_{0}\right)$.

Proof. Assume to the contrary that $z(\mathrm{t})$ satisfies $\left(\mathrm{N}_{0}\right)$. To simplify our notation, let us denote

$$
\mathrm{P}(\mathrm{t})=\pi(\mathrm{t}) \pi(\tau(\mathrm{t})) \mathrm{p}(\mathrm{t}) .
$$

An integration of $\left(E_{c}\right)$ from $s$ to $t$ yields

$$
r_{2}(s) \pi(s)\left(r_{1}(s) \pi(s) z^{\prime}(s)\right)^{\prime} \geqslant \int_{s}^{t} P(x) z(\tau(x)) d x \geqslant z(\tau(t)) \int_{s}^{t} P(x) d x
$$

Integrating twice from $s$ to $t$, one gets

$$
z(s) \geqslant z(\tau(t)) \int_{s}^{t} \frac{1}{r_{1}(v) \pi(v)} \int_{v}^{t} \frac{1}{r_{2}(v) \pi(v)} \int_{x}^{t} P(x) d v d u d x
$$

which setting $s=\tau(t)$ contradicts to $\left(C_{0}\right)$. 
Theorem 2.4. Let (2.1) holds. Assume that $\mathrm{y}(\mathrm{t})$ is a positive solution of $(\mathrm{E})$. If

$$
\liminf _{t \rightarrow \infty} \int_{\tau(t)}^{t} \pi(x) \pi(\tau(x)) p(x) \int_{t_{1}}^{x} \frac{1}{r_{1}(v) \pi(v)} \int_{t_{1}}^{v} \frac{1}{r_{2}(s) \pi(s)} \mathrm{d} s \mathrm{~d} v \mathrm{~d} x>\frac{1}{\mathrm{e}^{\prime}}
$$

then the corresponding $z(t)=y(t) / \pi(t)$ does not satisfy $\left(N_{2}\right)$.

Proof. Assume to the contrary that $z(t)$ satisfies $\left(N_{2}\right)$. Since $r_{2}(t) \pi(t)\left(r_{1}(t) \pi(t) z^{\prime}(t)\right)^{\prime}$ is positive and decreasing, one can verify that

$$
r_{1}(t) \pi(t) z^{\prime}(t) \geqslant \int_{t_{1}}^{t} r_{2}(s) \pi(s)\left(r_{1}(s) \pi(t) z^{\prime}(s)\right)^{\prime} \frac{1}{r_{2}(s) \pi(s)} d s \geqslant r_{2}(t) \pi(t)\left(r_{1}(t) \pi(t) z^{\prime}(t)\right)^{\prime} \int_{t_{1}}^{t} \frac{1}{r_{2}(s) \pi(s)} d s .
$$

An integration from $t_{1}$ to $t$ provides

$$
z(t) \geqslant r_{2}(t) \pi(t)\left(r_{1}(t) \pi(t) z^{\prime}(t)\right)^{\prime} \int_{t_{1}}^{t} \frac{1}{r_{1}(v) \pi(v)} \int_{t_{1}}^{v} \frac{1}{r_{2}(s) \pi(s)} d v d s
$$

Setting into $\left(E_{c}\right)$, we see that $w(t)=r_{2}(t) \pi(t)\left(r_{1}(t) \pi(t) z^{\prime}(t)\right)^{\prime}$ is a positive solution of differential inequality

$$
w^{\prime}(t)+\left(P(t) \int_{t_{1}}^{\tau(t)} \frac{1}{r_{1}(v) \pi(v)} \int_{t_{1}}^{v} \frac{1}{r_{2}(s) \pi(s)} d v d s\right) w(\tau(t)) \leqslant 0,
$$

where we employed (2.3). On the other hand, by Theorem 2.1.1 in [7], inequality (2.4) has no eventually positive solution. This is a contradiction and the proof is complete.

Now we are prepared to introduce our main result, which is a simple consequence of Theorems 2.3 and 2.4.

Theorem 2.5. Let (2.1) holds. Assume that $\left(\mathrm{C}_{0}\right)$ and $\left(\mathrm{C}_{2}\right)$ hold. Then $(\mathrm{E})$ is oscillatory.

\section{Examples}

We support our results by couple of illustrative examples.

Example 3.1. Consider the strongly noncanonical differential equation

$$
\left(t^{\beta}\left(t^{\alpha} y^{\prime}(t)\right)^{\prime}\right)^{\prime}+\frac{a}{t^{3-\alpha-\beta}} y(\lambda t)=0,
$$

where $a>0, \alpha>1, \beta>1$, and $\lambda \in(0,1)$. Simple computation shows that (2.1) holds true. Conditions $\left(\mathrm{C}_{0}\right)$ and $\left(\mathrm{C}_{2}\right)$ reduce to

$$
\frac{\mathrm{a} \lambda^{2-\alpha-\beta}}{(\beta-1)(\alpha+\beta-2)}\left\{\ln \frac{1}{\lambda}-\frac{1}{\beta-1}\left[1-\lambda^{\beta-1}\right]-\frac{1}{\alpha-1}\left[1-\lambda^{\beta-1}\right]+\frac{\beta-1}{(\alpha-1)(\alpha+\beta-2)}\left[1-\lambda^{\alpha+\beta-1}\right]\right\}>1
$$

and

$$
\frac{\mathrm{a} \lambda^{2-\alpha-\beta}}{(\alpha-1)(\alpha+\beta-2)} \ln \frac{1}{\lambda}>\frac{1}{\mathrm{e}^{\prime}}
$$

respectively. By Theorem 2.5, equation $\left(E_{1}\right)$ is oscillatory provided that both above conditions are satisfied. Example 3.2. Consider the third order differential equation

$$
\left(t^{\alpha}\left(t^{\alpha} y^{\prime}(t)\right)^{\prime}\right)^{\prime}+\frac{a}{t^{1-2 \alpha}} y(\lambda t)=0,
$$

where $a>0, \alpha>1$, and $\lambda \in(0,1)$. It is easy to verify that now both conditions $\left(C_{0}\right)$ and $\left(C_{2}\right)$ can be replaced by one condition

$$
\frac{a \lambda^{2-2 \alpha}}{2(\alpha-1)^{2}}\left\{\ln \frac{1}{\lambda}-\frac{2}{\alpha-1}\left[1-\lambda^{\alpha-1}\right]+\frac{1}{2(\alpha-1)}\left[1-\lambda^{2(\alpha-1)}\right]\right\}>1
$$

which guarantees oscillation of $\left(E_{2}\right)$. 


\section{Summary}

In this paper, we provided easily verifiable oscillatory criterion for noncanonical differential equation. Our criterion is based on a canonical representation of considered equation and essentially simplifies examination of noncanonical equations.

\section{Acknowledgment}

The paper is supported by the grant project KEGA 035TUKE-4/2017.

\section{References}

[1] J. Džurina, Comparison theorems for nonlinear ODE's, Math. Slovaca, 42 (1992), 299-315. 1, 1

[2] P. Hartman, Disconjugate $n$-th order linear differential equations and principal solutions, Bull. Amer. Math. Soc., 74 (1968), 125-129.

[3] P. Hartman, Principal solutions of disconjugate n-th order linear differential equations, Amer. J. Math., 91 (1969), 306362.

[4] I. T. Kiguradze, T. A. Chanturia, Asymptotic Properties of Solutions of Nonautonomous Ordinary Differential Equations, Kluwer Acad. Publ., Dordrecht, (1993). 1

[5] T. Kusano, M. Naito, Comparison theorems for functional differential equations with deviating arguments, J. Math. Soc. Japan, 33 (1981), 509-532. 1

[6] A. Y. Levin, Non-oscillation of solutions of the equation $x^{(n)}+p_{1}(t) x^{(n-1)}+\cdots+p_{n}(t) x=0$, Uspekhi Mat. Nauk, 24 (1969), 43-49.

[7] G. S. Ladde, V. Lakshmikantham, B. G. Zhang, Oscillation Theory of Differential Equations with Deviating Arguments, Marcel Dekker, New York, (1987). 2

[8] G. Pólya, On the mean-value theorem corresponding to a given linear homogeneous differential equations, Trans. Amer. Math. Soc., 24 (1922), 312-324. 1

[9] W. F. Trench, Canonical forms and principal systems for general disconjugate equations, Trans. Amer. Math. Soc., 184 (1974), 319-327.

[10] D. Willett, Asymptotic behaviour of disconjugate n-th order differential equations, Canad. J. Math., 23 (1971), $293-314.1$ 